\title{
Research on Day-ahead Dispatch of Electricity- heat Integrated Energy System Based on Improved PSO Algorithm
}

\author{
Xuanyue Shuai ${ }^{*}$, Xiuli Wang, Kaiying Li, Bangyan Wang, Shengqi Yuan, and Liang \\ Zhang \\ School of Xi'an Jiaotong University, Xi'an, China
}

\begin{abstract}
With the increasingly serious environmental pollution, it is of great significance to construct a comprehensive day-ahead dispatch model of the integrated energy system. For the regional electricity-heat integrated energy system, firstly analyze the operating characteristics and dispatch costs of all units in the system, and then establish the day-ahead economic dispatch model of the system. Furthermore, an improved PSO algorithm is designed based on the idea of adaptive weight and genetic algorithm, and the appropriate algorithm is used to solve the work schedule of each unit in the system through a calculation example. Then the sensitivity analysis of the electric boiler capacity is carried out. Finally, the feasibility of the proposed model is verified through the results analysis, which provides a reference scheme for the electricity-heat integrated energy system including electric vehicles.
\end{abstract}

Keywords: electricity-heat integrated energy system, economic dispatch, improved PSO algorithm, electric vehicles.

\section{Introduction}

With the large-scale development of non-renewable energy sources such as coal and oil, human society is facing a crisis of environmental degradation ${ }^{[1]}$. In order to improve the utilization rate of energy, the proposal of the Energy Internet has aroused great concern around the world ${ }^{[2]}$. The integrated energy system can coordinate and optimize the production, transmission, conversion, and distribution of energy ${ }^{[3]}$.So far, many domestic scholars have conducted research on integrated energy systems. Literature[4] proposed a multi-objective optimal scheduling model for an electricity-heat integrated energy system that takes into account network transmission losses. Literature[5] studied the optimal allocation of energy resource in high-proportion renewable energy system. Literature[6] proposed a joint optimal dispatch model of electricity-gas-heat integrated energy system with user-side participation.

The following content is: the second part establishes the unit model of the electricityheat integrated energy system, and the third part determines the objective function and

*Corresponding author: sxysxy@stu.xjtu.edu.cn 
constraint conditions, the fourth part proposes the improved PSO algorithm, the fifth part is the analysis of a calculation example, and finally explains the conclusion of this article.

\section{Unit Modeling in Electricity-Heat Integrated Energy System}

The electricity-heat integrated energy system described in this paper consists of three units. The electricity-heat coupling unit includes the CHP units and an electric boiler, the renewable power generation unit includes wind turbines, and the electricity energy storage unit mainly has energy batteries and electric vehicles. The system information management figure is shown in Figure 1.

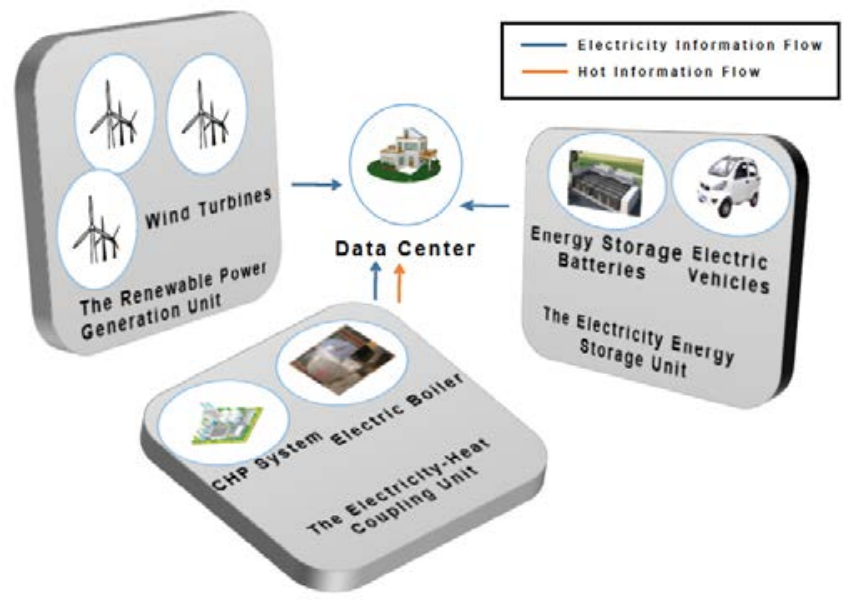

Figure 1. The system information management.

\subsection{CHP unit}

Assuming that a day can be divided into $\mathrm{H}$ time periods, the relationship between the fuel cost and output of the CHP unit in the time period $k$ of the day can be expressed as:

$$
C_{C H P}^{k}=c_{y} \sum_{i=1}^{n}\left(\frac{c_{e} P_{e, C H P, i}^{k}}{\eta_{e}}+\frac{277.78 c_{h} P_{h, C H P, i}^{k}}{\eta_{h}}\right) \Delta t
$$

In the formula, $k \in[1,2, \ldots, H]$ and $c_{\mathrm{y}}$ are the unit price of coal consumption, $\Delta t$ is the duration of each time period, and $P_{e, C H P, i}^{k}$ and $P_{h, C H P, i}^{k}$ are the electricity output and heat output of the CHP unit during the time period $k$.

At the same time, the electricity and heat output, ramp rate of the CHP unit at any $k$ period of the day shall meet:

$$
\begin{gathered}
P_{e, C H P(\min )}^{k} \leq P_{e, C H P}^{k} \leq P_{e, C H P(\max )}^{k} \\
P_{h, C H P(\min )}^{k} \leq P_{h, C H P}^{k} \leq P_{h, C H P(\max )}^{k} \\
\lambda_{e, C H P(\min )}^{k} \leq \frac{P_{e, C H P}^{k}-P_{e, C H P}^{k-1} \leq \lambda_{e, C H P(\max )}^{k}}{\Delta t}
\end{gathered}
$$




$$
\lambda_{h, C H P(\min )}^{k} \leq \frac{P_{h, C H P}^{k}-P_{h, C H P}^{k-1}}{\Delta t} \leq \lambda_{h, C H P(\max )}^{k}
$$

In the above four formulas, $P_{e, C H P(\min )}^{k}$ and $P_{e, C H P(\max )}^{k}$ respectively represent the minimum and maximum allowable electrical output of the CHP unit, and $P_{h, C H P(\min )}^{k}$ and $P_{h, C H P(\max )}^{k}$ respectively represent the minimum and maximum allowable thermal output of the CHP unit. $\lambda_{e, C H P(\min )}^{k}$ and $\lambda_{e, C H P(\max )}^{k}$ are the minimum and maximum value of the ramp rate of the CHP's electric power respectively, and $\lambda_{h, C H P(\min )}^{k}$ and $\lambda_{h, C H P(\max )}^{k}$ are the minimum and maximum value of the ramp rate of the CHP's thermal output respectively.

\subsection{Electric boiler}

Considering that the electric boiler itself does not consume additional primary energy, and assuming that factors such as the life cost of the electric boiler are not considered, the heat output of the electric boiler during the $k$ period of the day can be expressed as:

$$
P_{h, E B}^{k}=\eta_{E B} P_{e, E B}^{k}
$$

The heat output of the electric boiler in any $k$ time period of the day should meet:

$$
0 \leq P_{h, E B}^{k} \leq P_{h, E B(\max )}
$$

In the formula, $\eta_{E B}$ is the conversion efficiency of the electric boiler, $P_{e, E B}^{k}$ is the electric power consumed by the electric boiler in the $k$ period of the day, and $P_{h, E B(\max )}$ is the maximum allowable heat output of the electric boiler.

\subsection{Wind turbine}

Due to the reduction in the utilization rate of renewable energy due to wind curtailment, which affects the economic cost of the system, the wind curtailment cost of the wind turbine in the s-th period of the day can be expressed as:

$$
C_{W}^{k}= \begin{cases}c_{\mathrm{w}} \sum_{i=1}^{n_{\mathrm{w}}}\left[P_{w, i}^{k}-P_{W T, i}^{k}\right] \Delta t & \left(P_{w}^{k}-P_{W T}^{k}>0\right) \\ 0 & \left(P_{w}^{k}-P_{W T}^{k} \leq 0\right)\end{cases}
$$

In the formula, $c_{w}$ is the unit abandonment cost, $n_{w}$ is the total number of wind turbines in the system, and $P_{w}^{k}$ and $P_{W T}^{k}$ are the actual output and grid-connected power of the $i$ wind turbine in the $k$ period of the day.

\subsection{Energy storage battery}

In this paper, the battery is used as the energy storage battery, the total capacity of the battery in the $k$ period of the day can be expressed as: 


$$
E_{E S S}^{k}=(1-\delta) E_{E S S}^{k-1}+P_{c}^{k} \eta_{c} \Delta t-\frac{P_{d}^{k}}{\eta_{d}} \Delta t
$$

In the formula, $E_{E S S}^{k-1}$ is the remaining battery power at time $k-1$, unit: kwh; $\delta$ is the self-discharge power of the battery, unit: kw; $P_{c}$ and $P_{d}$ are battery charging power and discharging power, unit: $\mathrm{kw} ; \eta_{c}$ and $\eta_{d}$ are battery Charge and discharge efficiency.

Due to the loss of energy storage battery in the process of power transmission, the cost of battery transmission loss during the $k$ period of the day is:

$$
C_{E S S}^{k}=c_{E S S}\left[P_{c}^{k}\left(1-\eta_{c}\right) \Delta t-P_{d}^{k}\left(\frac{\eta_{d}-1}{\eta_{d}}\right) \Delta t\right]
$$

In the formula, $c_{E S S}$ is the unit power loss cost of the battery, unit: yuan/MWh.

Due to the constraints on the capacity of the energy storage battery and the charge and discharge power, the capacity of the energy storage device in any $k$ time period of the day should meet:

$$
S_{E(\min )} \leq E_{E S S}^{k} \leq S_{E(\max )}
$$

In the formula, $S_{E(\min )}$ and $S_{E(\max )}$ are the minimum capacity and maximum capacity allowed by the energy storage device, respectively.

The charging and discharging power of the energy storage device in any $k$ time period of the day shall meet:

$$
-P_{E, c(\max )} \leq P_{E}^{k} \leq P_{E, d(\max )}
$$

In the formula, $P_{E, d(\max )}$ and $P_{E, c(\max )}$ are the maximum allowable charging power and the maximum allowable discharging power of the energy storage device.

\subsection{Electric vehicle}

This article assumes that the user-side electric vehicle battery is used as the energy storage device, and the electric vehicle participates in the system scheduling during the non-driving period. Different from the energy storage battery, let $\Phi=\{8: 00-18: 00\}$ be the electric vehicle disable scheduling period, and the electric vehicle scheduling period needs to be restricted as follows:

$$
P_{E V}^{k}=0 \quad(k \in \Phi)
$$

\section{Objective Function and System Constraints}

The goal of the daily dispatch of the electricity-heat integrated energy system is to reasonably arrange the output of each unit in the system to meet the system's electricity and heat active power balance and the operating constraints of each unit, while minimizing the total daily dispatch cost of the system.

The total dispatch cost of the system in a day can be expressed as: 


$$
F=\sum_{k=1}^{H}\left(C_{C H P}^{k}+C_{W}^{k}+C_{E S S}^{k}+C_{E V}^{k}\right) \Delta t
$$

The equipment involved in adjusting the electrical load balance in the system mainly includes the CHP units, energy storage batteries, electric vehicles, fans, and electric boilers. Any $k$ time period of the day needs to meet:

$$
P_{E V}^{k}+P_{E S S}^{k}+P_{W}^{k}+P_{e, C H P}^{k}=P_{L}^{k}+P_{e, E B}^{k}
$$

In the formula, $P_{L}^{k}$ is the total electrical load of the system at any time period $k$ of the day, and $P_{E V}^{k}$ and $P_{E S S}^{k}$ are the discharge power of the electric vehicle battery and energy storage device at any time period $k$ of the day.

The equipment involved in adjusting the heat load balance in the system mainly includes the CHP units and electric boiler, so any $k$ time period of the day needs to meet:

$$
P_{h, C H P}^{k}+P_{h, E B}^{k}=P_{h}^{k}
$$

In the formula, $P_{h}^{k}$ is the total heat load of the system during any $k$ time period of the day.

\section{The Process of Improved PSO Algorithm}

The general PSO algorithm update process is shown in Figure 2:

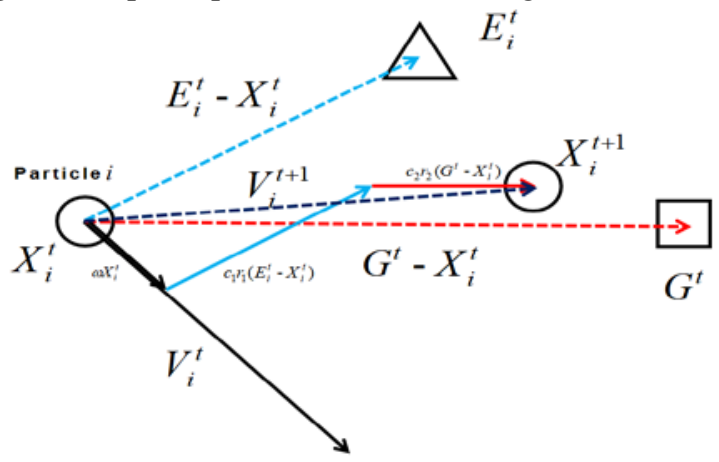

Figure 2. The general PSO algorithm update process.

This paper hybridizes the position and velocity of $\kappa$ particles in the particle swarm on the basis of genetic algorithm. Set the hybridization probability as $\psi$, the position and velocity of the $\kappa$ particles offspring in $h$ particle can be expressed as:

$$
\begin{array}{r}
X_{h}^{t}=r r_{1} X_{u}^{t}+\left(1-r r_{1}\right) X_{r}^{t} \\
V_{h}^{t}=r r_{1} V_{u}^{t}+r r_{1} V_{r}^{t} \cdot \frac{\mathrm{I}_{1}}{\mathrm{I}_{2}}
\end{array}
$$

In the formula, $\kappa \subseteq N, h \in[1,2, \ldots, \kappa] ; r r_{1}$ is a random number uniformly distributed from 0 to $1 ; u$ and $\kappa$ are the number of hybridized particles from A particles, respectively, 
and $u, r \in[1,2, \ldots, \kappa] ; \mathrm{I}_{1}$ and $\mathrm{I}_{2}$ are the maximum singular values of vectors $r r_{1} V_{u}^{t}$ and $r r_{1} V_{u}^{t}+r r_{1} V_{r}^{t}$, respectively.

Then $r r_{2}$ and $r r_{3}$ can be further expressed as:

$$
\begin{gathered}
u=\left\lfloor r r_{2}(\kappa-1)\right\rfloor+1 \\
r=\left\lfloor r r_{3}(\kappa-1)\right\rfloor+1
\end{gathered}
$$

In the formula, Both $u$ and $r$ are random numbers uniformly distributed from 0-1.

At the same time, the inertia weight of the $i$ particle in the $t$ iteration is dynamically adjusted as follows:

$$
\omega_{i}^{t}= \begin{cases}\omega_{(\min )}+\left(X_{i}^{t}-X_{(\min )}^{t}\right) \cdot \frac{\omega_{(\max )}-\omega_{(\min )}}{\sum_{i=1}^{N} X_{i}^{t}-X_{(\min )}^{t}}\left(E_{i}^{t}<\frac{1}{N} \sum_{i=1}^{N} E_{i}^{t}\right) \\ \omega_{(\max )} & \left(E_{i}^{t} \geq \frac{1}{N} \sum_{i=1}^{N} E_{i}^{t}\right)\end{cases}
$$

In the formula, $\omega_{(\min )}$ and $\omega_{(\max )}$ respectively represent the minimum and maximum values of the set inertia weight.

\section{Case Analysis}

Take a certain area in northern of China as an example. The area is equipped with 60 distributed wind turbines with a rated capacity of 2MW, 1 electric boiler, 200 energy storage batteries, 200 electric vehicle batteries and 4 CHP units. All CHP units work in the mode of fixed electricity by heat. The rated capacity of the batteries and electric vehicles battery in the system are $96 \times 200 \mathrm{kwh}$ and $50 \times 200 \mathrm{kwh}$, and the maximum charge and discharge power are $78 \times 200 \mathrm{kw}$ and $40 \times 200 \mathrm{kw}$, respectively. The $c_{y}$ is 500 yuan/ton, and $c_{w}$ is 500 yuan/ton, and the rated power and output limits of CHP, wind turbine and electric boiler are shown in Table 1, and the remaining relevant parameters of the CHP are shown in Table 2. Let $\mathrm{H}=24$, and electricity load, heat load and wind power forecast curve of the electricity-heat integrated energy system in a day are shown in Figure 3(a).

Table 1. The rated power and output limits of CHP, wind turbines and electric boiler.

\begin{tabular}{|c|c|c|c|}
\hline Object & $\begin{array}{c}\text { Rated } \\
\text { power/MW }\end{array}$ & $\begin{array}{c}\text { Minimum output } \\
\text { value/MW }\end{array}$ & $\begin{array}{c}\text { Maximum output } \\
\text { value/MW }\end{array}$ \\
\hline CHP & 350 & 175 & 350 \\
\hline wind turbine & 2 & 0 & 2 \\
\hline electric boiler & 20 & 0 & 20 \\
\hline
\end{tabular}

Table 2. The remaining relevant parameters of the CHP

\begin{tabular}{|c|c|c|c|c|c|c|c|}
\hline Parameter & $\eta_{e}$ & $\eta_{h}$ & $c_{e}$ & $c_{h}$ & $\theta$ & $\lambda_{\min }$ & $\lambda_{\text {max }}$ \\
\hline Value & 0.354 & 0.893 & 0.1288 & 0.123 & 1 & $-100 \mathrm{MW} / \mathrm{h}$ & $100 \mathrm{MW} / \mathrm{h}$ \\
\hline
\end{tabular}




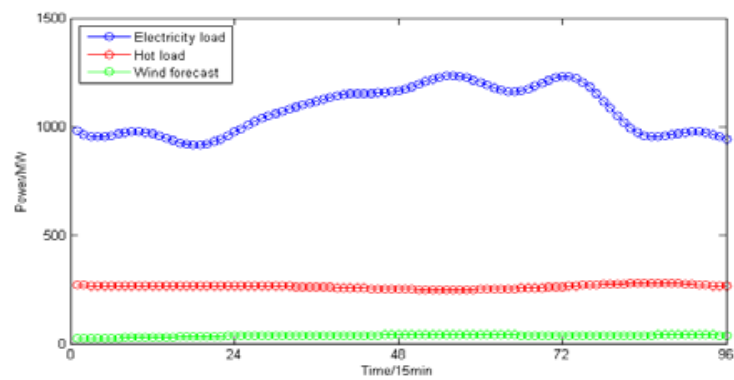

(a)

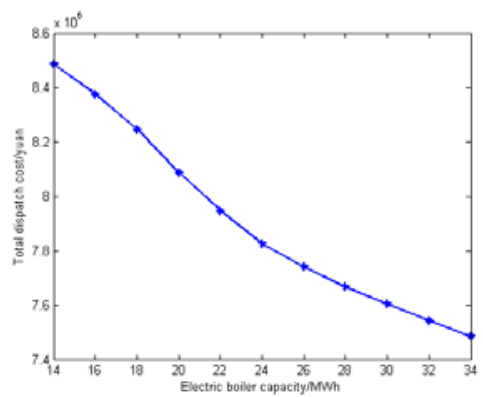

(b)

Figure 3. (a) Electricity load, heat load and wind power forecast curve

(b) Relationship curve between total system dispatch cost and electric boiler capacity.

Suppose that only the CHP is considered as mode 1 in the system, only the CHP and electric boiler are considered as mode 2 in the system, and all units are considered as mode 3 , and the number of iterations of the improved PSO algorithm is set to 1000 . The cost of system scheduling in the three modes is shown in Table 3.

Table 3. The rated power and output limits of CHP, wind turbines and electric boiler.

\begin{tabular}{|c|c|c|c|c|c|}
\hline mode & $\begin{array}{c}\text { CHP coal } \\
\text { cost/ten } \\
\text { thousand yuan }\end{array}$ & $\begin{array}{c}\text { Wind } \\
\text { curtailment } \\
\text { cost/ten } \\
\text { thousand yuan }\end{array}$ & $\begin{array}{c}\text { Energy storage } \\
\text { battery dispatch } \\
\text { cost/ten } \\
\text { thousand yuan }\end{array}$ & $\begin{array}{c}\text { Electric vehicle } \\
\text { dispatch cost/ten } \\
\text { thousand yuan }\end{array}$ & $\begin{array}{c}\text { Total cost/ten } \\
\text { thousand yuan }\end{array}$ \\
\hline 1 & 733.50 & 175.70 & 0 & 0 & 909.20 \\
\hline 2 & 675.50 & 129.27 & 0 & 0 & 820.01 \\
\hline 3 & 674.83 & 123.63 & 6.02 & 3.06 & 807.54 \\
\hline
\end{tabular}

It can be seen from the above table that there are only CHP units in Mode 1, and the system is forced to abandon wind. In the abandonment mode 2, the electric boiler realizes electric and thermal coupling, which significantly consumes wind power resources. The introduction of storage batteries and electric vehicles in Mode 3 realizes the temporal and spatial transfer of electric energy and further absorbs wind power resources.

In the three modes, CHP electric output, electric boiler output are shown in Figure 4 (a) and (b), and the charging and discharging power curve of battery and electric vehicle in mode 3 is shown in Figure 4(c). Under the three modes, the charging and discharging power curve of batteries and electric vehicles and the curtailment curve are shown in Figure 3 (c) and (d).

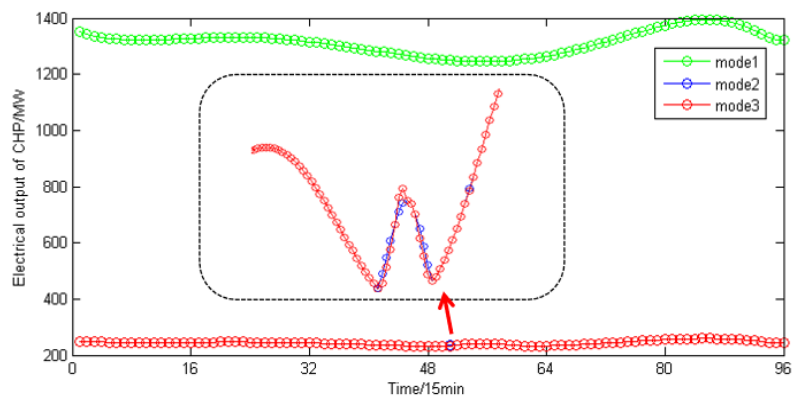

(a) 


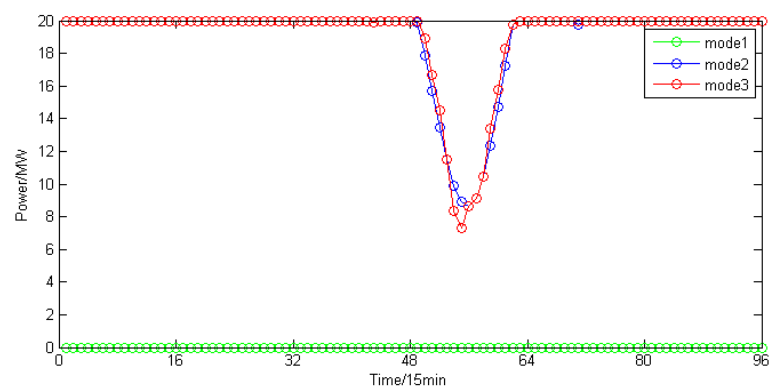

(b)

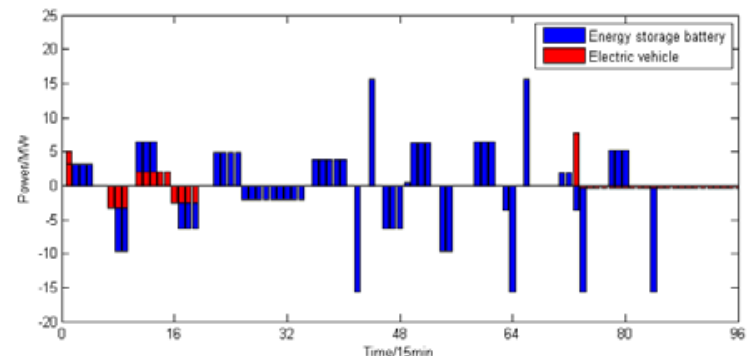

(c)

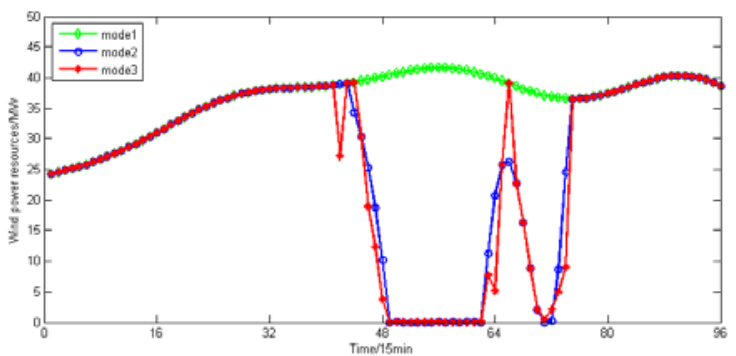

(d)

Figure 4. Model solution specific results.

In order to further explore the influence of electric boiler capacity on the total cost of system dispatch, set the electric boiler capacity from $14 \mathrm{MWh}$ to $34 \mathrm{MW}$, and the curve obtained is shown in Figure 3(b). This allows the operator to make a trade-off between the purchase cost of the electric boiler and the reduction in the total cost of dispatching, and select the optimal purchase plan.

\section{Conclusions}

1) The economic dispatch model of the electric-heat integrated energy system proposed in this paper provides a feasible optimization plan for the system containing electric vehicles, which can significantly improve the economic benefits of the system.

2) The proposed improved PSO algorithm makes full use of the advantages of adaptive weights and genetic algorithms to ensure that the particles are prevented from falling into the local optimum and the solution efficiency is low.

3) The influence of the maximum heat output of the electric boiler on the total cost of the system is additionally studied, which provides a reference plan for the system to choose the electric boiler model. 


\section{Acknowledgments}

This work was financially supported by the project of Yangzhong Green Energy Island Planning Reanalysis and Prospect Research.

\section{References}

1. Dong Chaoyang, Zhao Junhua, Wen Fushuan, etc. From Smart Grid to Energy Internet: Basic Concepts and Research Framework [J]. Automation of Electric Power Systems, 2014.

2. Mohsenian-Rad H. Coordinated price-maker operation of large energy storage units in nodal energy markets [J]. IEEE Trans on Power Systems, 2016, 31(1): 786-797.

3. Wang Yingrui. Economic dispatch of electricity-heat-gas integrated energy system based on conditional value-at-risk[D]. North China Electric Power University (Beijing), 2017.

4. Zheng Chaoming, Huang Bonan, Wang Zixin, etc. Multi-objective optimal dispatch of electric-heat integrated energy system considering network transmission loss $[\mathrm{J}]$. Power System Technology, 2020(01):144-157.

5. Lu Xiaomin, Chen Lei, Xu Fei, etc. Research on the optimal ratio of power supply capacity of West Mengxi Power Grid under the framework of electric-heat integrated energy system[J]. Electric Power Automation Equipment, 2017, 37(06): 132-138.

6. Deng Minghui, Jiang Yunsong, Yan Keke, etc. Optimal dispatch of integrated energy system with the participation of power users[J]. Journal of Electric Power System and Automation, 2019, 31(05): 49-56. 